\title{
Divulgando en píldoras y desarrollando competencias orales y escritas en el alumnado de Cálculo Numérico*
}

\author{
Luis M. Abia ${ }^{1}$, M. Paz Calvo ${ }^{2}$, Begoña Cano ${ }^{3}$, Paula M. López-Pérez ${ }^{4}$ \\ Departamento de Matemática Aplicada, Facultad de Ciencias e IMUVA, \\ Universidad de Valladolid, Campus Miguel Delibes, Paseo Belén 7, 47011, Valladolid \\ ${ }^{1}$ abia@mac.uva.es, ${ }^{2}$ mariapaz.calvo@uva.es, ${ }^{3}$ bego@mac.uva.es, \\ ${ }^{4}$ paulamaria.lopez@uva.es
}

\begin{abstract}
This paper presents the teaching innovation project which has been carried out on the subject Numerical Methods, belonging to the first year of the Mathematics Degree. The aim of this project is twofold. On the one hand, it has been intended to provide the students with some key competencies, basic for the Degree and for their future career, like speaking skills, analysing and presenting results skills, and writing skills. In order to acquire these tools, students were proposed to create scientific posters, which were presented at the end of the academic year in a special workshop. On the other hand, the second goal of the project has been to reveal students some practical applications, in Physics and Engineering, of the numerical methods studied on the subject. In order to do that, five knowledge pills have been created, all of them available on the YouTube channel UVa_Online.
\end{abstract}

Keywords: Knowledge pills, speaking competencies, ${ }^{A} T_{E} X$, writing competencies, Kahoot!

\section{Resumen}

En este trabajo se presenta el proyecto de innovación docente que se ha llevado a cabo en la asignatura Cálculo Numérico de Primer Curso del Grado en Matemáticas. El objetivo de este proyecto es doble. Por un lado se ha pretendido que el alumnado adquiera varias competencias básicas tanto para el Grado como para su futura vida laboral, como son la comunicación oral, la capacidad de analizar y presentar resultados, y la comunicación escrita. Para adquirir dichas competencias, entre otras actividades, se ha organizado una jornada de pósteres al final de curso. Por otro lado, se

\footnotetext{
${ }^{*}$ Proyecto financiado por la Universidad de Valladolid
} 
Divulgando en píldoras y desarrollando competencias orales y escritas en el alumnado de Cálculo Numérico

ha tenido como propósito que el alumnado conozca aplicaciones de la vida real, en la física y la ingeniería, de los métodos numéricos estudiados en la asignatura. Para ello el profesorado que forma parte del proyecto ha creado cinco píldoras de conocimiento, disponibles en el canal UVa_Online de YouTube.

Keywords: Pildoras de conocimiento, competencias orales, ${ }^{A} T_{E} X$, competencias escritas, cálculo numérico, Kahoot!

\section{Introducción}

En el marco del Espacio Europeo de Educación Superior (EEES) se apunta a la educación por competencias (RIesco, M. 2015; Zabalza, M. 2005; Del Carmen Leví Orta, G. And Ramos MÉndez, E. 2013). El término competencia significa, conceptualmente, "habilidad compleja e integrada de llevar a cabo una determinada tarea" (LAsnier, F. 2000). Por lo tanto, el profesorado debe diseñar actividades que generen situaciones de aprendizaje centradas en el desarrollo de la capacidad del estudiantado de resolver problemas, que deberían reproducir situaciones de la vida real (Vilaplana, R. and Gomis, O. and Hyder, A. 2010). No sólo deben desarrollarse competencias específicas de cada asignatura, en este caso Cálculo Numérico, sino también otras más generales (Instituto DE CiEnCiAS DE LA EduCACión 2006). En este caso, el proyecto de innovación docente se ha centrado tanto en comunicación oral (Universitat Politècnica de VAlÈncia 2018) como en comunicación escrita, además de en la capacidad de analizar y presentar resultados. Al mismo tiempo, se considera imprescindible animar al alumnado a la interacción y al trabajo con sus semejantes (PÉrez de Albéniz Iturriaga, A. 2015), por lo que se les ha pedido que trabajen por grupos en distintas actividades, que serán especificadas en el apartado Desarrollo de la innovación.

La utilización de material audiovisual online, en concreto de píldoras de conocimiento, comienza a constituirse un nuevo paradigma del contexto educativo EEES (GUtiérrez San Miguel, B. and Rodríguez Fidalgo, M. I. 2015). Sande Mayo, M. J. 2014 resalta que "La posibilidad de que el alumnado pueda reproducir cuantas veces considere necesario el contenido del soporte digital hasta su completa comprensión, presenta una clara ventaja en relación con las lecciones presenciales, en las que las explicaciones del o la docente, muy a a pesar del alumnado, no cuentan con la opción de rebobinado". En este escenario se enmarca otro de los propósitos de este trabajo, que el alumnado conozca aplicaciones de la vida real, en la física y la ingeniería, de los métodos numéricos estudiados en la asignatura, por medio de la visualización de varias píldoras de conocimiento, creadas específicamente por miembros del proyecto. Se ha considerado necesario puesto que en general, el estudiantado no tiene una percepción clara de la relación que existe entre lo que aprende en clase y la aplicación práctica que la materia tiene en la vida real, cuando es indudable que son muchas las aplicaciones del cálculo numérico en problemas de la física y la ingeniería (Kumar, S. And Jalkio, J. A. 1999). Se desarrolla la metodología en el apartado Desarrollo de la innovación.

La adecuación al EEES ha supuesto que la motivación por aprender se haga más necesaria que nunca. Experiencias previas con la gamificación han demostrado un aumento de la motivación intrínseca de los estudiantes por la asignatura y un aumento 
en el número de aprobados. El uso de la herramienta Kahoot! en diferentes ámbitos educativos ha sido estudiado previamente y siempre se han obtenido resultados muy positivos (Iwamoto, D. H. and Hargis, J. and Taitano, E. J. and Vuong, K. 2017) . Las investigaciones concluyen que Kahoot! es una buena herramienta para realizar actividades en el aula, contribuye a mejorar la participación del alumnado fomentando una relación positiva entre el grupo de alumnos y alumnas, aumenta la tasa de asistencia a clase y el alumnado lo percibe como un juego (SEMPERE FERRE, F. 2018). Por todo ello, en este trabajo se ha utilizado esta herramienta de cara a la evaluación de la comprensión por parte del alumnado de las píldoras de conocimiento creadas. Se exponen los resultados obtenidos en el apartado Resultados.

El EEES introduce además una metodología docente basada en proyectos, presentada formalmente por William Heart Kilpatrick en 1918. Este profesor de la Universidad de Columbia planteaba que "El aprendizaje se produce de mejor manera cuando es consecuencia de experiencias significativas, ya que esto permite al estudiantado coparticipar en la planificación, producción y comprensión de una experiencia" (KILPATRICK, J. W. 1968). Bajo esta metodología, se ha organizado una jornada de pósteres, en la que el alumnado ha puesto en práctica cada una de las competencias que se pretendía que adquirieran.

El proyecto de innovación docente descrito en el presente trabajo ha sido aplicado al alumnado del Grado en Matemáticas, del Programa de estudios conjunto de Grado en Física y Grado en Matemáticas, y del Programa de estudios conjunto de Grado en Ingeniería Informática de Servicios y Aplicaciones y Grado en Matemáticas de la Universidad de Valladolid. En concreto al estudiantado de primero, de la asignatura cuatrimestral de Cálculo Numérico (6 créditos ECTS), en particular durante las clases de prácticas. En esta asignatura hay 60 estudiantes, que se dividen en dos grupos.

\section{Objetivos}

Se plantean los siguientes objetivos, que encajarían en el modelo educativo del Espacio Europeo de Educación Superior (EEES):

- Desarrollar competencias de comunicación oral en el alumnado, aprendiendo herramientas básicas para hablar en público y poniéndolas en práctica.

- Desarrollar competencias de comunicación escrita en el alumnado, aprendiendo a utilizar el sistema de composición de texto $\mathrm{AT}_{\mathrm{E}} \mathrm{X}$.

- Divulgar conocimiento por medio de píldoras formativas (útiles tanto para el alumnado como para otros u otras docentes). Se mostrarán algunas aplicaciones prácticas, en problemas reales de la física y la ingeniería, de la materia que estudian en clase.

- Conseguir que el alumnado aprenda a analizar y exponer los resultados de sus prácticas. 


\section{Desarrollo de la innovación}

Se ha observado que, en general, el estudiantado no tiene una percepción clara de la relación que existe entre lo que aprende en clase y la aplicación práctica que la materia tiene en la vida real, cuando es indudable que son muchas las aplicaciones del cálculo numérico en problemas de la física y la ingeniería. Además de eso, el alumnado no tiene prácticamente ocasiones para desarrollar sus competencias de comunicación oral hasta que se enfrenta a la preparación de su Trabajo Fin de Grado, ni tampoco para aprender el sistema de composición de textos $\mathrm{LT}_{\mathrm{E} X}$, tan necesario en la creación de artículos, libros y pósteres científicos. Es por ello que en este trabajo se propone proveer al alumnado de herramientas básicas para hablar en público y saber exponer su trabajo, además de acercarle algunas aplicaciones reales del cálculo numérico.

Por un lado, se han creado cinco píldoras de conocimiento para que el estudiantado las visualice antes de realizar cada una de las prácticas a entregar. Estas píldoras han sido grabadas en el plató del servicio de medios audiovisuales de la Universidad de Valladolid por una docente y una investigadora que forman parte del proyecto. En cada una de ellas se explica la idea básica de la práctica que el alumnado va a desarrollar y un ejemplo de la vida real (en física, ingeniería u otros campos) en el que se utilizan esos métodos numéricos. Dichos ejemplos han sido escogidos cuidadosamente por el personal docente e investigador que forma parte del proyecto.

Por otro lado, también se ha propuesto que cada estudiante exponga durante cinco minutos algún contenido relacionado con la asignatura. Lo han hecho en diferentes días (al empezar o terminar cada una de las clases de prácticas) y sobre distintos temas (algunas personas han contado a la clase qué entendieron después de ver la píldora, otras han explicado los resultados obtenidos una vez realizada la práctica y cómo llegaron a ellos, etc.)

Por último, también se ha organizado una jornada de divulgación, donde el alumnado ha presentado cada una de las prácticas que han hecho durante el curso y su aplicación a problemas de la vida real, por medio de pósteres que se han encargado de preparar y presentar las y los estudiantes mismos (siendo la plantilla en $\mathrm{AT}_{\mathrm{E} X} \mathrm{X}$ para la realización de dichos pósteres facilitada por los y las docentes y la investigadora que forman parte del proyecto, para no cargar de trabajo en exceso al alumnado).

Además de todo esto, se han impartido dos charlas. Una de ellas ha tenido como finalidad que aprendan herramientas básicas de comunicación oral, siendo esta conferencia impartida por un experto en la materia, quién se encarga además de impartir el curso de formación al profesorado Hablar en público y habilidades sociales de la Universidad de Valladolid. La otra charla ha girado en torno a matemática aplicada , cálculo numérico y simulación numérica, siendo impartida por una investigadora que forma parte del proyecto, miembro de la Sociedad Española de Matemática Aplicada (SEMA).

Especificamos a continuación cada una de las actividades realizadas. 


\subsection{Creación de píldoras de conocimiento}

De cara a hacer llegar al alumnado la aplicación práctica de los métodos numéricos aprendidos en clase, se han creado cinco píldoras de conocimiento. Cada una de ellas está ligada a una de las cinco prácticas a entregar por el alumnado. Mediante reuniones periódicas del equipo de trabajo, se fue dando forma al tema de cada una de ellas. Dado que la materia se divide en cinco grandes bloques, se han escogido los temas de las píldoras y las prácticas asociadas en el marco de alguno de ellos. A continuación se presentan los títulos de dichos bloques:

\section{Interpolación polinómica}

2. Cuadratura numérica

3. Derivación numérica

4. Ecuaciones no lineales

5. Solución de sistemas lineales

La primera píldora (ABIA, L. M. AND LóPEz-PÉREz, P. M. 2019), titulada Números de condición de un polinomio, se enmarca en el primer bloque. En ella se explican varios conceptos relacionados con el número de condición de un polinomio, tanto al evaluarlo en un punto como al hallar sus raíces. Se habla del efecto de la aritmética coma flotante, de cómo afecta la base que se utiliza para el cálculo del número de condición, de que la base de Bernstein (Farouki, R. T. and Googman, T. N. T. 1996) es la que produce números de condición menores, y se ilustran estos conceptos con el polinomio pérfido. Al final del mini-vídeo se plantea la práctica (realizada en MATLAB) que tendrán que entregar.

En la segunda píldora (LóPEz-PÉREz, P. M. 2019), titulada Interpolación y enmarcada también en el primer bloque, se ha pretendido mostrar dos aplicaciones prácticas de la interpolación que se explica en clase. Una de ellas es la interpolación de Lagrange, por lo que se hace mención de la figura del matemático Joseph Louis Lagrange, siendo también importante que el alumnado sepa situar en la historia a los y las matemáticas que han creado los métodos que estudia. Después de una pequeña introducción al concepto de elementos finitos (Ciarlet, P. G. 2002), se les dan dos ejemplos prácticos como son la propagación de ondas submarinas en relación a conocer la batimetría del fondo marino, y la propagación de ondas en una tubería bicapa, de forma que se puedan localizar defectos en su interfaz (LóPEz-PÉREz, P. M. 2017). Finalmente, se presenta la práctica que tendrán que realizar.

Enmarcada en el segundo bloque, la tercera píldora (ABIA, L. M. AND CANo, B. 2019), titulada Cuadratura Adaptativa, trata de acercar el concepto de cuadratura adaptativa, explicándose cómo aproximar la integral definida

$$
\int_{0}^{\infty} x \cos e^{x} \mathrm{~d} x
$$

con diez cifras significativas correctas (Bornemann, F. And Laurie, D. And WaGON, S. AND WALdvogel, J. 2004). Para ello, se describen reglas de cuadratura simples (de los trapecios, del punto medio y de Simpson) y compuestas, junto a sus 


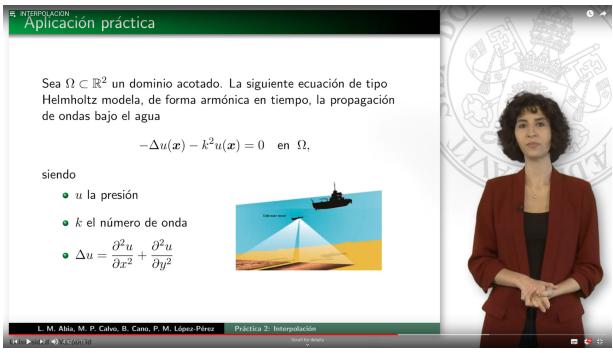

Fig. 1: Escena correspondiente a la segunda píldora grabada.

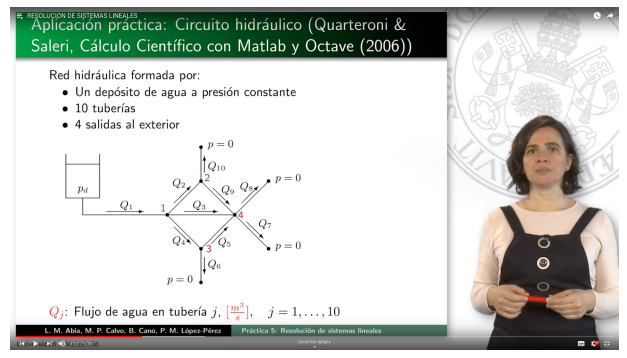

Fig. 2: Escena correspondiente a la quinta píldora grabada.

errores, además de un algoritmo adaptativo (Moler, C. B. 2004) utilizado para resolver el problema planteado. En este marco, se plantea la práctica a realizar.

Para ilustrar que los métodos numéricos pueden ser aplicados a la resolución de problemas de la vida diaria, la cuarta píldora (CAlvo, M. P. And López-PÉrez, P. M. 2019), titulada Resolución de ecuaciones no lineales y enmarcada en el cuarto bloque, plantea el problema de decidir si es posible desplazar, deslizando por el suelo, un armario por un pasillo con una distribución y anchura como las que se muestran en la Figura 3 (Quarteroni, A. And Saleri, F. 2003). Este problema se resuelve utilizando el método de Newton estudiado en clase (SANZ-SERnA, J. M. 2010). La práctica a entregar gira también en torno a dicho método.

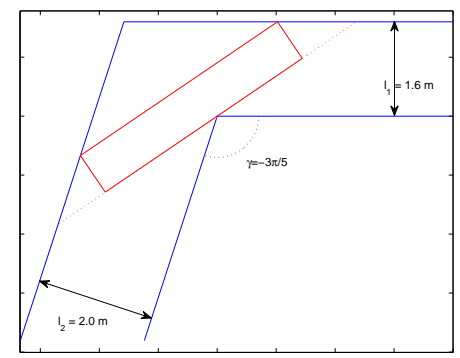

Fig. 3: Situación del pasillo por el que se quiere deslizar un armario.

La resolución de sistemas lineales es el tema de la quinta y última píldora (CANO, B. 2019), titulada Resolución de sistemas lineales y enmarcada en el quinto bloque. En ella se presenta un problema concreto: averiguar los flujos de agua en las distintas tuberías de una red hidráulica (Quarteroni, A. And Saleri, F. 2003), cuya resolución requiere resolver un sistema lineal. Para ello, se utilizará un algoritmo de eliminación gaussiana.

Notar que todas las píldoras han sido grabadas en el plató del servicio de medios audiovisuales de la Universidad de Valladolid, con el formato "Saber, extender" (Figura 4). Las Figuras 1 y 2 muestran escenas correspondientes a dos de las píldoras creadas.

Además de la grabación de estas píldoras, la investigadora postdoctoral Paula M. López Pérez ha impartido una charla titulada Simulación numérica y métodos numéri$c o s$, donde se ha explicado someramente en qué consiste una simulación numérica y 


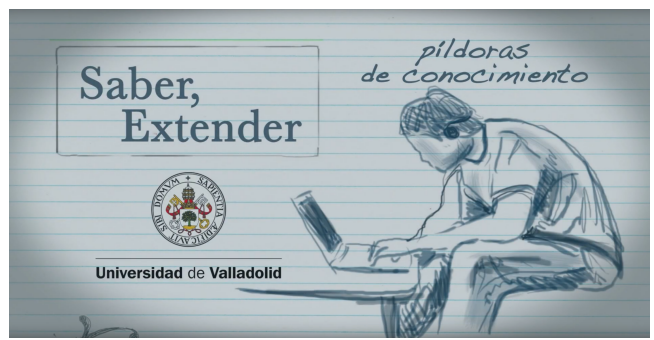

Fig. 4: Formato en el que se publican las píldoras de conocimiento.

en qué punto de ella se aplican los métodos numéricos aprendidos en clase, ilustrando esto con distintos ejemplos de aplicaciones en Física e Ingeniería en los que el Cálculo Numérico proporciona una herramienta esencial para la resolución de los problemas que se plantean.

\subsection{Desarrollo de competencias orales}

El primer paso para mejorar las capacidades de comunicación oral del alumnado ha sido darles herramientas básicas para hablar en público. Para ello, el conferenciante Lucas Burgueño, que también se encarga de impartir en la Universidad de Valladolid cursos de formación al profesorado, les ha impartido una charla. En ella han escuchado y puesto en práctica conceptos básicos para la comunicación oral.

En segundo lugar, cada estudiante ha tenido la oportunidad de hacer una breve exposición oral en las clases de prácticas de la asignatura. Parte del alumnado ha presentado un resumen de la píldora que se les había pedido visualizar esa semana, y otra parte ha explicado cómo realizó la práctica que estaba asociada a dicha píldora y ha analizado los resultados obtenidos.

Por último, a final de curso ha tenido lugar una Jornada de pósteres, donde el alumnado ha tenido ocasión de presentar ante profesorado, alumnado y público en general el póster que habían realizado, explicando tanto los métodos numéricos utilizados como los resultados obtenidos.

\subsection{Desarrollo de competencias escritas}

En primer lugar se ha preparado una plantilla básica en $\mathrm{HT}_{\mathrm{E}} \mathrm{X}$ de un póster. Se ha intentado hacerla lo más sencilla posible, para no cargar de trabajo en exceso al alumnado, que está en su primer año de Grado. A continuación se les ha hecho llegar la plantilla en $\mathrm{IAT}_{\mathrm{E}} \mathrm{X}$ al alumnado, para que se familiaricen con dicho sistema de composición de textos. Más tarde, reunidos en grupos de tres o cuatro personas, han utilizado la plantilla para realizar un póster sobre una de las cinco píldoras y su práctica asociada que se trabajan durante el cuatrimestre. En total se han realizado 15 pósteres, uno por cada grupo. Por último, a final de curso han defendido sus trabajos ante el público en la Jornada de pósteres. En la Figura 5 se expone un ejemplo de dos pósteres realizados por el alumnado. 
Divulgando en píldoras y desarrollando competencias orales y escritas en el alumnado de
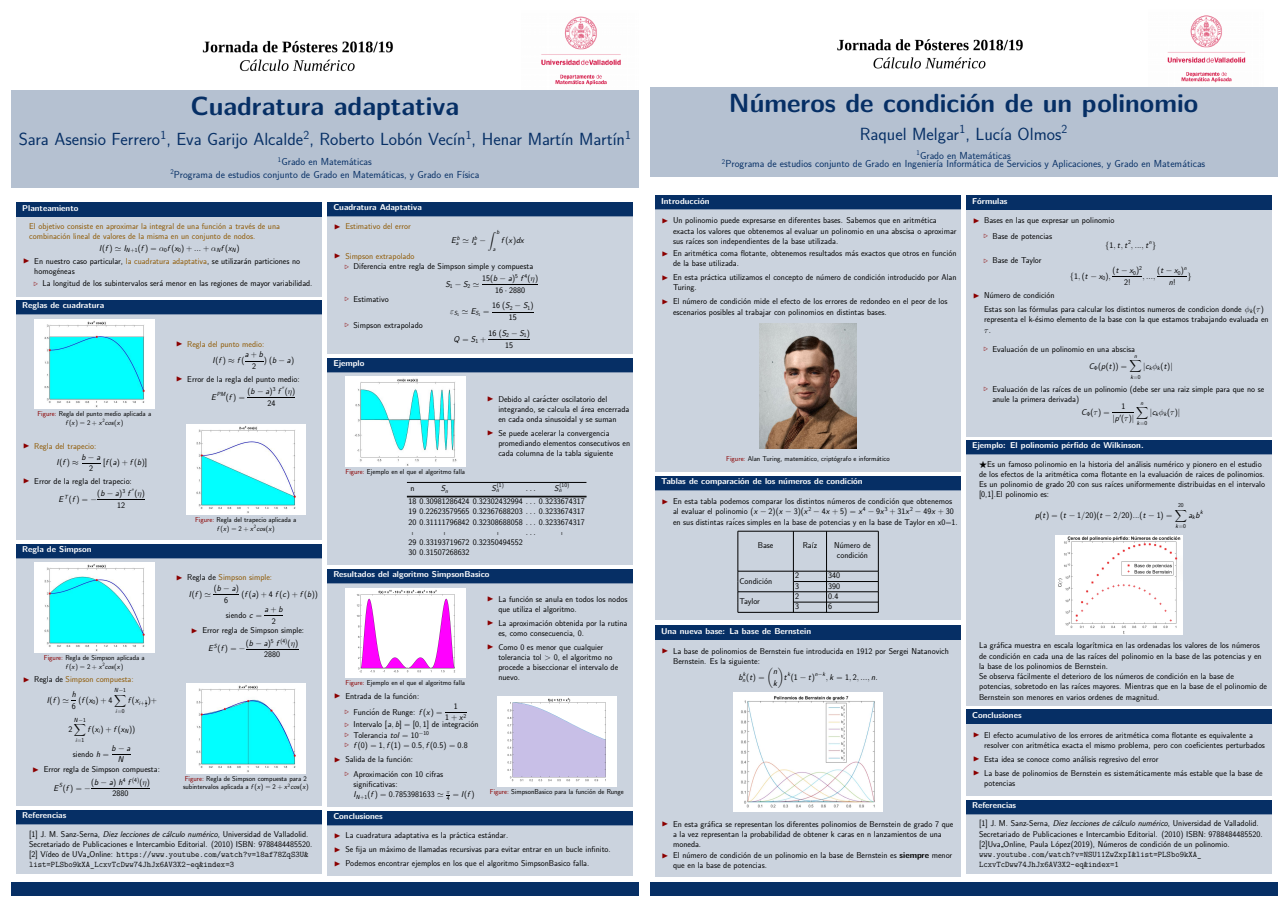

Fig. 5: Ejemplo de dos pósteres realizados por el alumnado, presentados en la Jornada de pósteres.

\section{Resultados}

Tres meses después de que se subieran las píldoras al canal UVa_Online de YouTube, el número de visitas a las píldoras es el siguiente:

\begin{tabular}{|c|c|c|c|c|}
\hline Píldora 1 & Píldora 2 & Píldora 3 & Píldora 4 & Píldora 5 \\
\hline \hline 570 & 317 & 362 & 206 & 219 \\
\hline
\end{tabular}

De entre este número de visitas, notar que un $60 \%$ de las visualizaciones fueron hechas desde un ordenador y un $30 \%$ desde un teléfono móvil.

Transcurrida una semana desde que el alumnado tuvo acceso a la primera píldora, se hizo en clase un cuestionario de cinco preguntas acerca del contenido de la misma. Se utilizó para ello la herramienta Kahoot! Respondieron al cuestionario 49 estudiantes. En la Figura 6 puede verse el número de estudiantes que respondieron de forma correcta e incorrecta a cada una de las cinco preguntas.

De forma análoga, se utilizó la herramienta Kahoot! para plantear al alumnado un cuestionario de cinco preguntas acerca del contenido de la segunda píldora. En este caso respondieron al cuestionario 44 estudiantes. En la Figura 7 puede verse el número de estudiantes que respondieron de forma correcta e incorrecta a cada una de las cinco preguntas. 


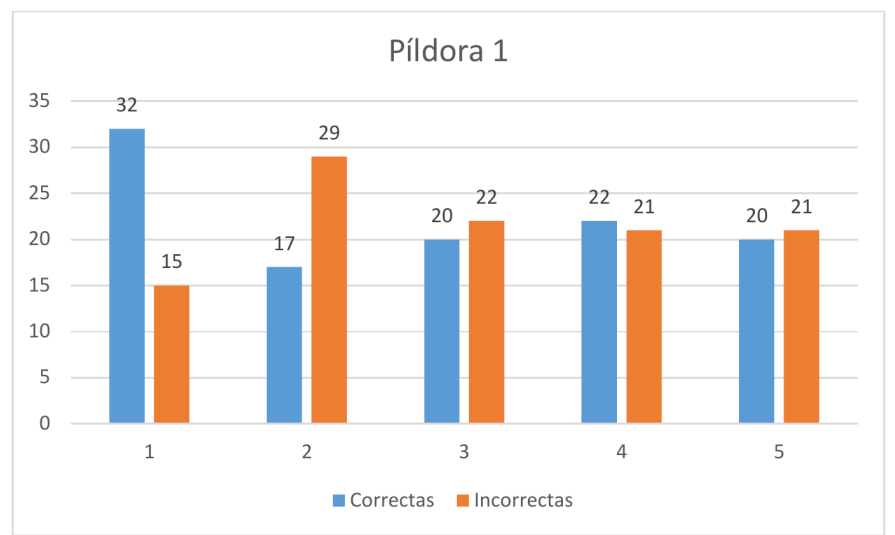

Fig. 6: Número de respuestas correctas e incorrectas a cada pregunta del cuestionario realizado sobre la Píldora 1.

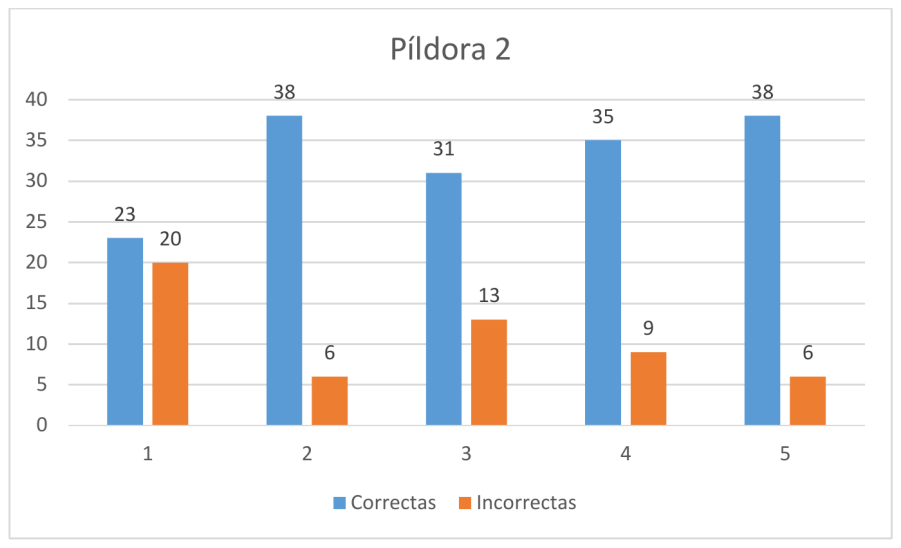

Fig. 7: Número de respuestas correctas e incorrectas a cada pregunta del cuestionario realizado sobre la Píldora 2.

El cuestionario sobre la tercera píldora fue respondida por 39 estudiantes, pudiendo verse en la Figura 8 el número de personas que respondieron de forma correcta e incorrecta a cada una de las cinco preguntas. Análogamente, los resultados del cuestionario sobre la cuarta píldora, contestado por 25 estudiantes, pueden verse en la Figura 9.

Por último, el cuestionario sobre la quinta y última píldora fue respondida por 35 estudiantes, pudiendo verse en la Figura 10 el número de personas que respondieron de forma correcta e incorrecta a cada una de las cinco preguntas.

A continuación se exponen los resultados obtenidos en la encuesta de satisfacción realizada al alumnado. A la fecha de cierre de este artículo, se tenían 16 respuestas.

En la Figura 11 pueden verse la valoración, del 1 al 5, siendo 1 en total desacuerdo y 5 totalmente de acuerdo, que se hace sobre el uso de la herramienta Kahoot!. En el diagrama de la izquierda se responde a la pregunta "¿El Kahoot! ha mejorado 
Divulgando en pildoras y desarrollando competencias orales y escritas en el alumnado de Cálculo Numérico

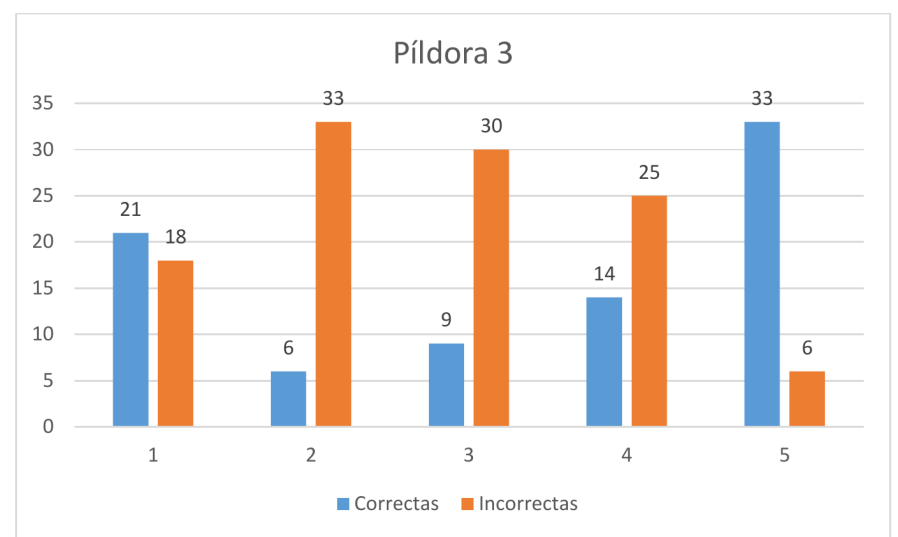

Fig. 8: Número de respuestas correctas e incorrectas a cada pregunta del cuestionario realizado sobre la Píldora 3.

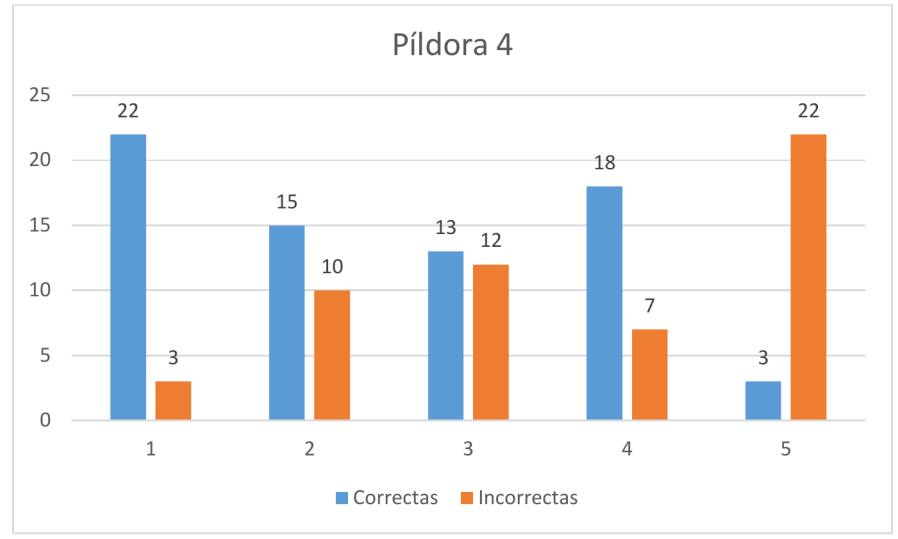

Fig. 9: Número de respuestas correctas e incorrectas a cada pregunta del cuestionario realizado sobre la Píldora 4.

tu motivación en clase?"Puede verse que el $65 \%$ del alumnado que ha respondido está entre muy y totalmente de acuerdo con esta afirmación. En el diagrama central se responde a la pregunta "¿El Kahoot! te ha parecido divertido?", a lo que más del $66 \%$ responde muy positivamente. Por último, en el diagrama de la derecha se responde a la pregunta "¿Te parece apropiado el uso del Kahoot! como valoración de tu comprensión del contenido de las píldoras?", siendo en este caso un $72 \%$ de las personas encuestadas las que están entre muy y algo de acuerdo.

En la Figura 12 se representa la valoración del 1 al 5 , siendo 1 en total desacuerdo y 5 totalmente de acuerdo, de la pregunta "¿Te ha servido esta píldora para conocer mejor aplicaciones prácticas del cálculo numérico?", para cada una de las cinco píldoras. Puede verse que, en general, han visto clara la aplicación práctica de los métodos que estudian en clase.

La siguiente pregunta trata sobre la utilidad para su futuro de las competencias puestas en práctica. En la Figura 13 puede verse, en el diagrama de la izquierda, 


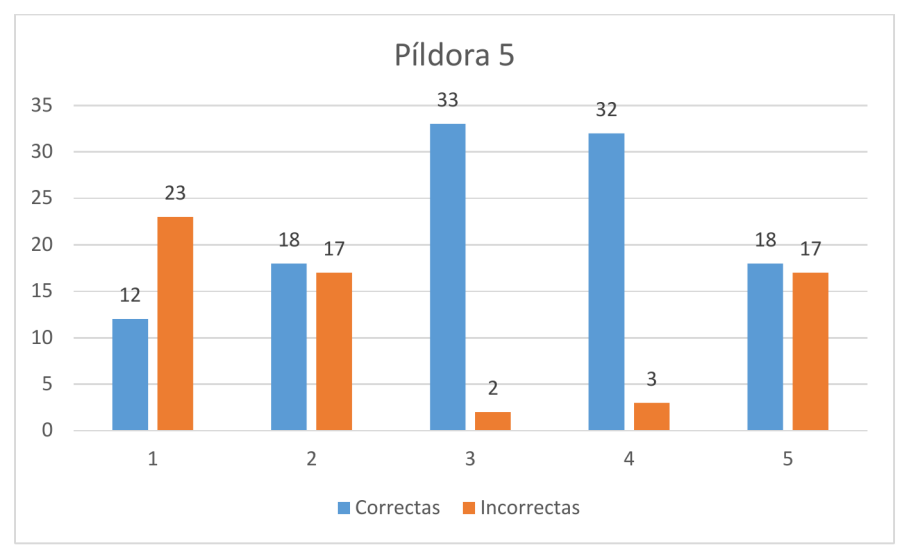

Fig. 10: Número de respuestas correctas e incorrectas a cada pregunta del cuestionario realizado sobre la Píldora 5.

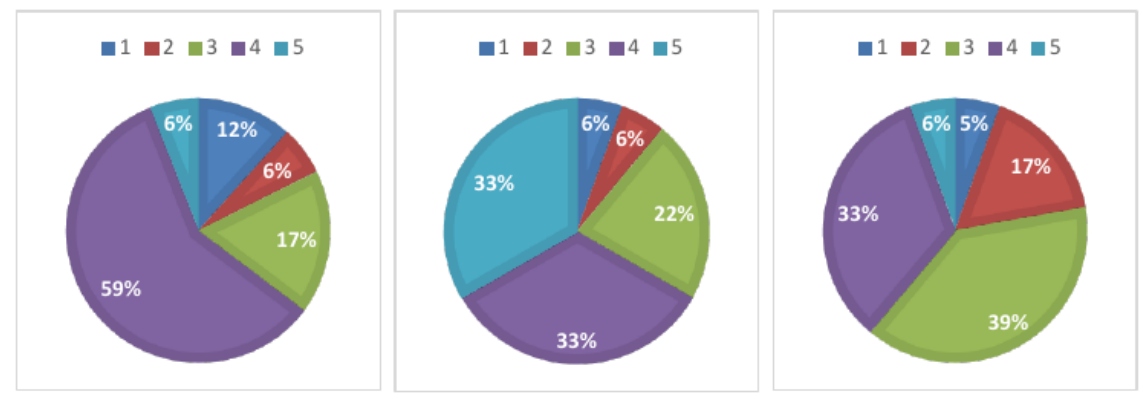

Fig. 11: Valoración del 1 al 5, siendo 1 en total desacuerdo y 5 totalmente de acuerdo de varias preguntas relacionadas con el uso de la herramienta Kahoot!: a la izquierda se les pregunta si ha mejorado su motivación en clase, en el centro si ha sido divertido y a la derecha si les ha parecido una herramienta adecuada.

su valoración del 1 al 5, de forma análoga a diagramas anteriores, de la utilidad de mejorar su comunicación oral. En el diagrama central sobre la utilidad de aprender LATEX, y en el diagrama de la derecha sobre la Jornada de pósteres. Puede verse que, su bajo su percepción, son las habilidades de comunicación oral las más útiles para su futuro.

Es relevante evaluar si este proyecto de innovación docente es un complemento a la asignatura Cálculo Numérico o les ha llevado más horas de las deseadas, por lo que en la Figura 14 se presenta el resultado de evaluar el número de horas dedicadas a realizar los pósteres. Puede verse que la mayoría del alumnado encuestado no ha dedicado más de siete horas, con lo que el hecho de haberles facilitado una plantilla ha hecho que no tuvieran una carga excesiva de trabajo.

Finalmente, se ha evaluado el grado de satisfacción global de las actividades adicionales que han realizado en el marco de este proyecto, pudiendo verse en la Figura 15 que casi el $90 \%$ del alumnado encuestado está entre algo y muy satisfecho. 
Divulgando en píldoras y desarrollando competencias orales y escritas en el alumnado de Cálculo Numérico

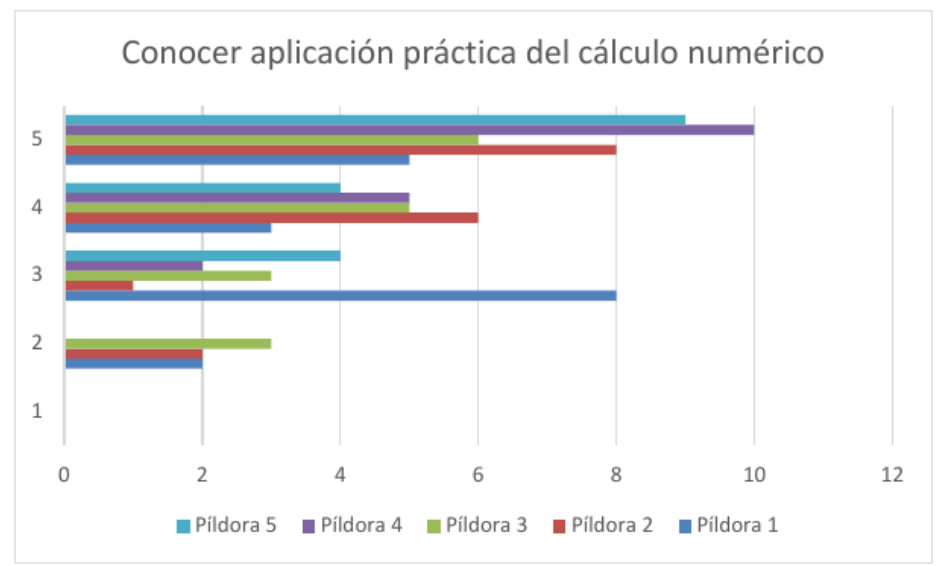

Fig. 12: Valoración del 1 al 5, siendo 1 en total desacuerdo y 5 totalmente de acuerdo de cuánto les ha servido cada píldora para conocer aplicaciones prácticas del cálculo numérico.
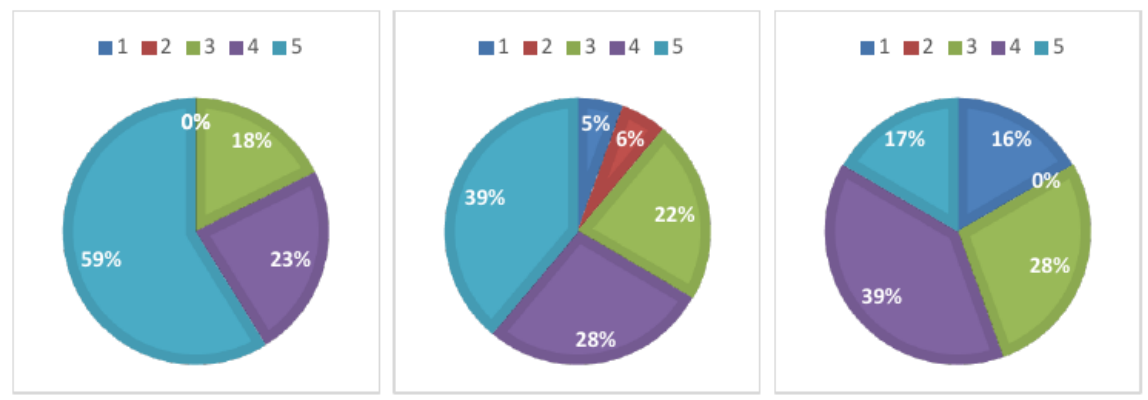

Fig. 13: Valoración del 1 al 5, siendo 1 en total desacuerdo y 5 totalmente de acuerdo de varias preguntas relacionadas con la utilidad futura de las competencias puestas en práctica: a la izquierda se les pregunta por comunicación oral, en el centro por el aprendizaje de IATEX, y a la derecha por la Jornada de pósteres.

\section{Agradecimientos}

Este trabajo ha sido financiado parcialmente por la Convocatoria de Proyectos de Innovación Docente de la Universidad de Valladolid para el curso 2018-2019, en concreto al PID $n^{\circ} 19$.

\section{Conclusiones}

En este trabajo se ha presentado el proyecto de innovación docente llevado a cabo durante el curso 2018/19 en la asignatura Cálculo Numérico de primer curso del Grado en Matemáticas. Se ha pretendido desarrollar competencias de comunicación oral (aprendiendo herramientas básicas para hablar en público y poniéndolas en práctica) y escrita (aprendiendo a utilizar el sistema de composición de textos $\mathrm{AT}_{\mathrm{EX}} \mathrm{X}$ para la realización de pósteres) en el alumnado, además de divulgar conocimiento por medio 


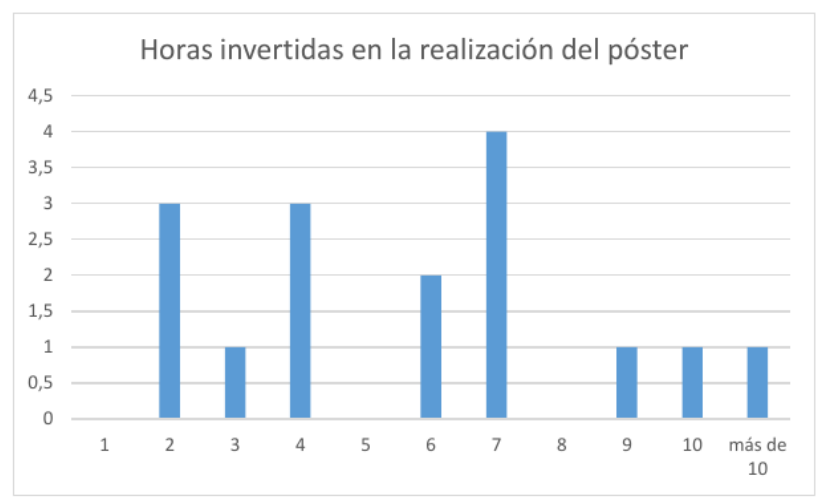

Fig. 14: Número de horas invertidas en la realización del póster, siendo el eje de las ordenadas el número de personas que dedican esas horas.

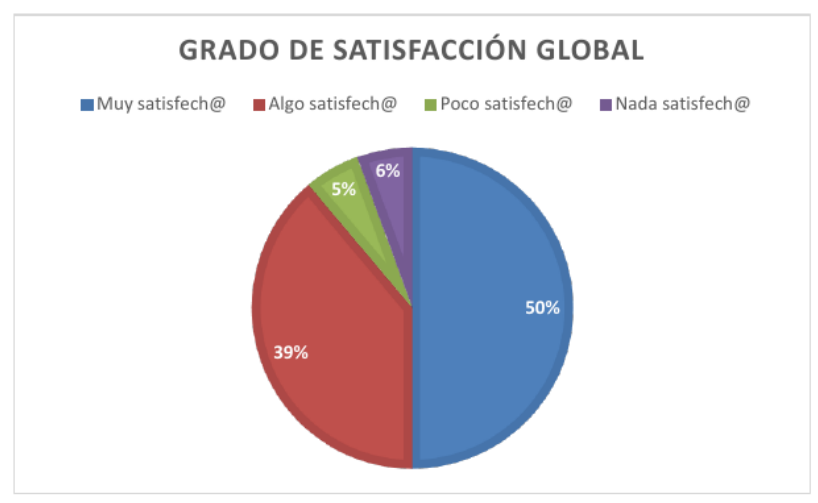

Fig. 15: Grado de satisfacción alcanzado con las actividades adicionales.

de píldoras formativas (útiles tanto para el alumnado como para otros/as docentes), mostrando algunas aplicaciones prácticas, en problemas reales de la física y la ingeniería, de la materia estudiada. Se han planteado los objetivos perseguidos en el proyecto, y las acciones que se han llevado a cabo para alcanzarlos. Se han descrito brevemente los contenidos de las píldoras que se han grabado y las actividades dirigidas a desarrollar competencias orales y escritas en el alumnado. Por último, se han mostrado resultados que ilustran el interés y el grado de comprensión que el alumnado ha alcanzado tras visualizar las píldoras y trabajar en las prácticas que en ellas se proponen, además de evaluar su grado de satisfacción y su percepción de utilidad con respecto a las actividades adicionales llevadas a cabo. 
Divulgando en pildoras y desarrollando competencias orales y escritas en el alumnado de

\section{Referencias bibliográficas}

Abia, L. M. And CAno, B. (2019). Cuadratura adaptativa. UVa_Online: Aplicaciones prácticas de métodos numéricos. URL: https://www . youtube. $\operatorname{com} /$ watch? $\mathrm{v}=$ 18af78ZqS3U\&list=PLSbo9kXA_LcxvTcDww74JhJx6AV3X2-eq\&index=3.

Abia, L. M. AND López-PÉRez, P. M. (2019). Números de condición de un polinomio. UVa_Online: Aplicaciones prácticas de métodos numéricos. URL: https:// www . youtube. com/watch?v=NSU11ZwZxpI\&list=PLSbo9kXA_LcxvTcDww74JhJx6 AV3X2-eq\&index $=1$.

Bornemann, F. and Laurie, D. and Wagon, S. and Waldvogel, J. (2004). The SIAM 100-digit challenge. A study in high-accuracy numerical computing. SIAM. ISBN: 089871561X.

Calvo, M. P. And López-PÉrez, P. M. (2019). Resolución de ecuaciones no lineales. UVa_Online: Aplicaciones prácticas de métodos numéricos. URL: https: // www . youtube. com/watch?v=WVbD7QylprM\&list=PLSbo9kXA_LcxvTcDww74JhJx$6 \mathrm{AV} 3 \mathrm{X} 2$-eq\&index $=4$.

Cano, B. (2019). Resolución de sistemas lineales. UVa_Online: Aplicaciones prácticas de métodos numéricos. URL: https : //www . youtube. com/watch?v=-9BeHo5cCo\&list=PLSbo9kXA_LcxvTcDww74JhJx6AV3X2-eq\&index $=5$.

Ciarlet, P. G. (2002). The finite element method for elliptic problems. Vol. 40. SIAM. ISBN: 0898715148.

Del Carmen Leví Orta, G. and Ramos Méndez, E. (2013). "Componentes de las competencias en los nuevos grados de algunas universidades españolas". En: Revista de Educación , 362 .

Farouki, R. T. and Googman, T. N. T. (1996). "On the optimal stability of the Bernstein basis". En: Mathematics of Computation Vol. 65. , 216, págs. 1553-1566.

Gutiérrez San Miguel, B. And Rodríguez Fidalgo, M. I. (2015). "Elaboración de píldoras del conocimiento al servicio de la divulgación científica". En: Revista de ciencias humanas y sociales ,2, págs. 593-609. ISSN: 1012-1587.

Instituto De Ciencias de la Educación (2006). Plan de acción para la convergencia europea. Guía docente. Universidad politécnica de Valencia.

Iwamoto, D. H. and Hargis, J. and Taitano, E. J. and Vuong, K. (2017). "Analyzing the efficacy of the testing effect using Kahoot on student performance”. En: Turquish Online Journal of Distance Education Vol. 18. , 2, págs. 80-93.

Kilpatrick, J. W. (1968). Una teoría de la nueva educación acomodada a nuestro tiempo. Biblioteca pedagógica. Editorial Losada. 
Kumar, S. AND JaLkiO, J. A. (1999). "Teaching mathematics from an applications perspective". En: Journal of Engineering Education Vol. 88, págs. 275-279. DOI: 10.1002/j.2168-9830.1999.tb00447.x.

LASNIER, F. (2000). Réussir la formation par competencies. Ed. Guérin. ISBN: 2760156982.

LóPEz-PÉREz, P. M. (2017). "Numerical study of time-harmonic acoustic problems in layered media using partition of unity finite element methods". Tesis doct. Universidade da Coruña.

- (2019). Interpolación. UVa_Online: Aplicaciones prácticas de métodos numéricos.

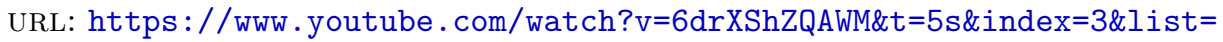
PLSbo9kXA_LcXvTcDww74JhJx6AV3X2-eq.

Moler, C. B. (2004). Numerical computing with MATLAB. SIAM. ISBN: 9780898715606.

PÉrez de Albéniz Iturriaga, A. (2015). "Metacognición en un proceso de aprendizaje autónomo y cooperativo en el aula universitaria". En: Contextos educativos: Revista de educación , 18.

Quarteroni, A. And SAleri, F. (2003). Scientific computing with MATLAB. Vol. 2. Springer-Verlag. ISBN: 3540443630.

RIEsco, M. (2015). "El enfoque por competencias en el EEES y sus implicaciones en la enseñanza y el aprendizaje." En: Tendencias Pedagógicas 13.0, págs. 79-106. ISSN: 1989-8614. URL: https : //revistas . uam .es/tendenciaspedagogicas / article/view/1892.

SAnde Mayo, M. J. (2014). "Una medicina para el conocimiento. Las píldoras educativas como recurso en la docencia del Derecho procesal". En: Reduca. Serie Derecho Procesal Vol. 5., 1, págs. 388-398.

Sanz-Serna, J. M. (2010). Diez lecciones de cálculo numérico. Universidad de Valladolid. Secretariado de Publicaciones e Intercambio Editorial. ISBN: 9788484485520.

Sempere Ferre, F. (2018). "Kahoot como herramienta de autoevaluación en la universidad". En: Congreso In-Red (2018), págs. 250-255.

Universitat Politècnica de VAlÈncia (2018). La comunicación efectiva como competencia transversal. URL: https://excelcon.blogs. upv.es/2015/02/24/ la-comunicacion-efectiva-como-competencia-transversal.

Vilaplana, R. And Gomis, O. And Hyder, A. (2010). "Working in Terms of Competences: Activities Designed Using Active Methodologies". En: New Achievements in Technology Education and Development. ISBN: 978-953-307-066-7. DOI: $10.5772 / 9220$.

Zabalza, M. (2005). Guía para la planificación didáctica de la docencia universitaria en el marco del EEES. Universidad de Santiago de Compostela. 